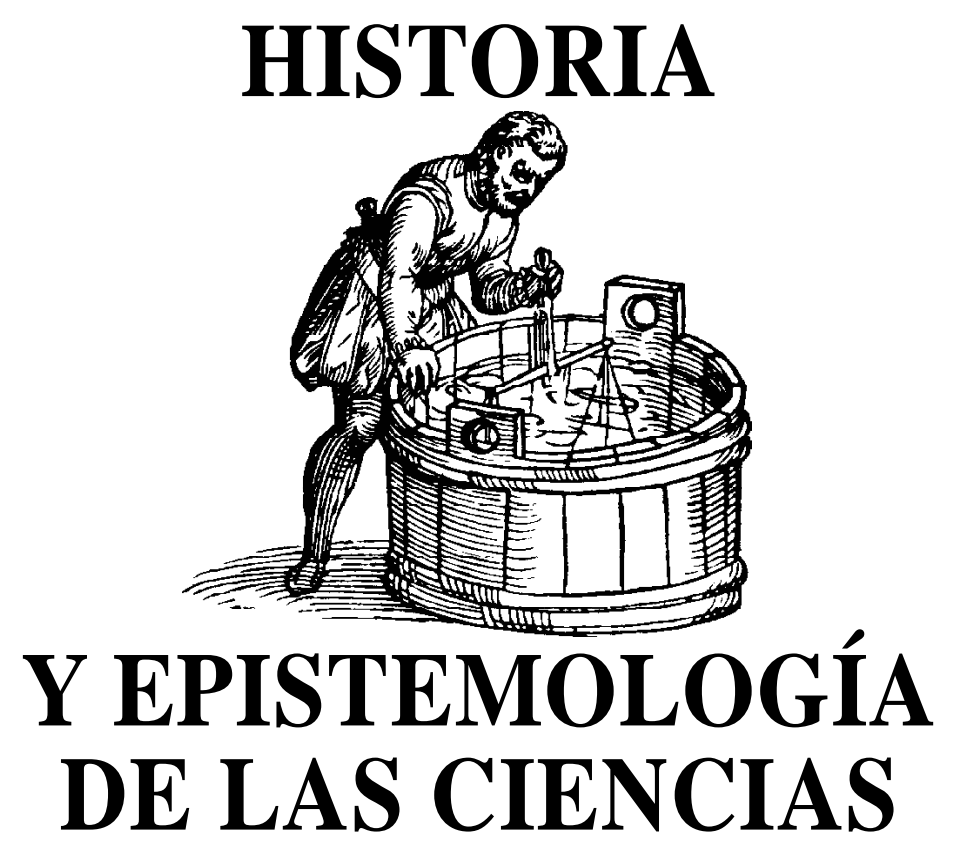

\title{
«PESAR» LA TIERRA: TEST NEWTONIANO Y ORIGEN DE UN ANACRONISMO
}

\author{
MORENO GONZÁLEZ, ANTONIO \\ Departamento de Didáctica de las Ciencias Experimentales. Universidad Complutense de Madrid. \\ Rector Royo Villanova, s/n. 28040 Madrid. \\ E-mail: diciex@eumos.sim.ucm.es
}

\section{SUMMARY}

The measure $G$, the universal gravitational constant, is attributed to Henry Cavendish. Nevertheless, the intention of the English physicist was to measure the density of the earth, which at that time was necessary in order to decide between different theories about the composition of this planet. $G$ was measured much later.

In this article I will try to explain how Cavendish accomplished the famous experiment and what his results were. Likewise, I will consider the problems that can arise in the scientific training of students from maintaining anachronisms such as this.

Al amigo y profesor Alberto Galindo, de quien he aprendido a valorar el rigor científico.

En los libros de texto hemos aprendido, y así se continúa enseñando, que el físico inglés Henry Cavendish (1731-
1810) realizó un experimento legendario utilizando una balanza de torsión «para medir la constante $G$ de la 
gravitación universal». De aquello hace doscientos años, y hasta fechas recientes no se ha reparado, al menos no se ha difundido, que no fue ése el resultado del experimento de Cavendish, quien lo que realmente hizo fue determinar la densidad media de la Tierra. Quizá en los primeros textos de física del siglo XIX, cuando se inicia la formulación de la ley de la gravitación como una igualdad y no en forma proporcional tal como la enunciara Newton, no se tomaron demasiado en cuenta las auténticas medidas de Cavendish y, como quiera que, conocida la densidad de la Tierra, fácilmente se obtiene $G$, pudo preferirse atribuir el cálculo de $G$ al famoso experimento y así simplificar las explicaciones. Si ésta fuera la justificación y si hubiera constancia que así es, no habría por qué alarmarse, pero a juzgar por los múltiples libros consultados, pasados y actuales, no se precisa en qué momentos fueron hechas las medidas de ambas magnitudes - densidad de la Tierra y $G-$ y, por lo tanto, sí que debe corregirse el error. Al no aclarar cómo fueron las cosas, la confusión se ha convertido en doctrina, atribuyéndosele a Cavendish un interés por el cálculo de una constante universal que ni tuvo él ni en su tiempo pudo tener nadie, porque éste es un asunto relacionado con las unidades de medida y la homogeneidad de las leyes físicas que no se practica sistemáticamente en la física hasta bien mediado el siglo XIX, como consecuencia de la aplicación a las magnitudes físicas del concepto de dimensión (Palacios, 1964) introducido por Jean Batiste Fourier (1768-1830) en su Théorie analytique de la Chaleur (1822), en la que por primera vez se plantea la necesidad de utilizar sistemas de unidades apropiados en las ecuaciones asociadas a los fenómenos físicos; de manera que la gratuita atribución de la medida de $G$ a Cavendish es un auténtico anacronismo. De él pretendo ocuparme aquí hasta donde permite la documentaciónconsultada en esta indagación aún inconclusa.

\section{SUPOSICIONES, DUDAS Y CONTROVERSIAS}

Entre las publicaciones del siglo Xvil que ponían de manifiesto que algo nuevo se estaba gestando (Rossi, 1997), la más relevante es sin duda Philosophiae Naturalis Principia Mathematica de Isaac Newton (16421727), publicada por la Royal Society londinense en 1687 a expensas de Edmund Halley (1656-1742), convencido de la genialidad de aquel tratado que supuso la primera gran unificación de la física, acabando con los distingos metafísicos de los antiguos entre el tratamiento de los fenómenos celestes y los terrestres.

«Esta admirable obra -escribe Pierre Simon Laplace (1749 -1827)- contiene los gérmenes de todos los grandes descubrimientos que han sido hechos después sobre el sistema del mundo: la historia de su desarrollo por los sucesores de este gran geómetra sería a la vez el comentario más útil sobre su obra y la mejor guía para llegar a nuevos descubrimientos.» (Laplace, 1904).

Y en otra de sus precisas publicaciones sobre la ley de Newton, dice el astrónomo y matemático francés:
«No existe punto en la física de veracidad más incontestable y mejor demostrado, de acuerdo con la observación y el cálculo, que el de: «todos los cuerpos celestes gravitan los unos sobre los otros». Newton, autor de este descubrimiento, el más importante hecho jamás en la filosofía natural, encuentra que los movimientos observados de los planetas no pueden subsistir sin una tendencia hacia el Sol, proporcional a su masa, y recíproco al cuadrado de su distancia a ese astro» (Laplace, 1773).

La base de los Principia se asienta, por tanto, sobre los que entonces se consideraron efectos de atracción, referidos al cuerpo hacia el que otro es arrastrado, y de gravitación, respecto al cuerpo que es arrastrado; si de la gravitación terrestre se trataba, el término convenido fue gravedad. La atracción entre partículas de materia se denominó cohesión. Y aunque ni Newton ni muchos de sus epígonos hicieran hipótesis sobre las causas de tales fuerzas, prefiriendo dejar en manos de la voluntad divina los designios del mundo, sí intentaron medirlas. Dándose por hecho que gracias a la gravitación universal se mantenía el orden celeste -la kepleriana «armonía de las esferas»-se trataba de comprobar, y a ser posible medir, esa misma supuesta atracción gravitatoria entre objetos cotidianos o entre objetos y la misma Tierra, sobre todo para corroborar la todavía dudosa pero, a su vez, versátil ley inversa del cuadrado de la distancia, pronto asociada también a los fenómenos electrostáticos y a los magnéticos.

Entendida por Newton la atracción como acción a distancia, fundamento de su mecánica, supuso un serio reparo para aceptar la teoría de la gravitación por la comunidad científica de la Europa continental, más afecta al mecanicismo cartesiano, defensor de las acciones por contacto en un mundo donde - para René Descartes (1596-1650) y sus seguidores- no era concebible el vacío y en el que los torbellinos deléter, materia sutil que todo lo llenaba, transmitía cualquier interacción entre cuerpos distantes. La controversia suscitada por ambas visiones del mundo llegó a convertirse en un problema nacionalista, especialmente en Francia (Lafuente, Delgado, 1984a), donde la Academia de Ciencias, los salones ilustrados -sedes de exhibiciones y polémicas científicas- y el propio Estado demandaron una respuesta convincente para la causa de la atracción, aunque el mismo Newton hubiera desestimado ocuparse de tales causas, pero que a los ojos de los cartesianos convencidos de tener muy clara la explicación de las interacciones, la propuesta newtoniana estaba más próxima a las cualidades ocultas de las físicas aristotélica y medieval, o al menos de las causas metafísicas para explicar el movimiento, que de las sólidas y contrastables razones que ya se exigía al conocimiento científico.

A criterio de la Academia de Ciencias de París, la determinación de la figura de la Tierra podría ser concluyente para decidir entre las contrapuestas teorías cartesiana y newtoniana. Sobre la forma de la Tierra, Newton, en el teorema XVI acerca de que los ejes de los planetas son menores que los diámetros trazados perpendicularmente a los ejes (proposición XVIII) del tercer libro de los Principia, conjetura: 
«La igual gravitación de las partes situadas en todos los lados de los planetas les daría una forma esférica si no fuera por su revolución diurna en círculo. Ese movimiento circular hace que las partes que se alejan del eje pugnen por ascender cerca del ecuador; como consecuencia de ello, si la materia está en estado fluido, al ascender hacia el ecuador, aumentará allí los diámetros $\mathrm{y}$, al descender hacia los polos, acortará el eje. Por tanto, el diámetro de Júpiter (por observaciones coincidentes de los astrónomos) resulta ser más corto entre polo y polo que de este a oeste. Y, a tenor de la misma argumentación, si nuestra Tierra no fuera más alta en el ecuador que en los polos, el mar se hundiría en las proximidades de los polos y, elevándose hacia el ecuador, anegaría cuanto allí hubiera» (Newton, 1982).

Combinado el principio de atracción con la medida de la fuerza centrífuga establecida por el holandés Christiaan Huygens (1629-1695) en su Horologium oscillatorium (1673), Newton postula que la Tierra es un esferoide con un achatamiento en los polos respecto al ecuador como 229 es a 230. Para comparar la curvatura de la superficie terrestre en lugares de distinta latitud se procedió a la medida de las respectivas longitudes de un grado de meridiano en dichos lugares, dedicándose a tal empresa grandes esfuerzos (Todhunter, 1873). Si la Tierra fuera esférica, el radio de curvatura del arco de meridiano, cualquiera que fuese el punto elegido, sería el mismo, el radio de la Tierra; las diferencias en la longitud del grado de meridiano, es decir, del arco cuyas latitudes extremas difiriesen un grado, sería tanto mayor cuanto menor fuese la curvatura, o sea, cuanto más achatada fuese la zona considerada (Taton, 1988).

Por otra parte, a lo largo del siglo XVIII, las controversias sobre la constitución de la Tierra se polarizaron en torno a dos posturas, tan radicalmente defendidas que incluso pusieron en peligro la credibilidad en la incipiente geología como ciencia de la naturaleza; disputas tan vehementes como las sostenidas entre antiguos y modernos o clásicos y románticos. El prusiano Abraham Gottlob Werner (1750-1817) consideraba que el océano era la fuente de todas las formaciones terrestres; la acción física y química del agua era el fundamento del reino mineral. A esta teoría neptuniana se opuso la plutoniana o volcanista del escocés James Hutton (1726-1797) que atribuía al fuego o calor central las formaciones geológicas básicas de la Tierra. Aunque su obra (Hutton, 1795) fue poco apreciada en su tiempo, la labor de sus seguidores, especialmente John Playfair (1748 -1819), autor de Illustrations of the Huttonian theory (1802), y la adhesión de los alemanes Alexander von Humbolt y Leopold von Buch, consiguió implantar las teorías de Hutton sobre las de Werner, gracias también al apoyo dispensado desde la recién creada Sociedad Geológica de Londres en 1807, que optó por los plutonianos si bien reconociendo la inexorable acción geológica del agua. El espaldarazo definitivo al plutonismo lo dio el geólogo escocés Charles Lyell (1797-1875) en The Principles of Geology: being an attempt to explain the former changes of the Earth's surface, by reference to causes now in action (1830-1833), escrito bajo la influencia de la síntesis newtoniana y con idénticas aspiraciones unificadoras a las expuestas en los Principia (Bowker, 1995).

Si la medida del arco de meridiano se aceptó para dilucidar la forma de la Tierra, medir su densidad fue la tarea emprendida para decidir sobre la fluidez o solidez del planeta, intentos ambos asociados a la admisión o no de la supuesta atracción newtoniana.

\section{A LA BÚSQUEDA DE SOLUCIONES: TANTEOS Y REPETICIONES}

La primera operación geodésica patrocinada por la Academia de Ciencias de París fue la medida de un grado de latitud entre Malvoisine y Sourdon realizada por el astrónomo y abate Jean Picard (1620-1682), colaborador de Gassendi en sus comienzos, autor de La mesure de la Terre (París, 1671), donde da un valor de $39.801 \mathrm{~km}$ para la circunferencia terrestre, contándose entre los primeros en reparar que la variación, con la latitud, de la longitud del péndulo que bate segundos podía servir para la determinación de la figura de la Tierra. En la primera edición de los Principia, Newton utilizó el valor del grado de latitud de Picard. En 1722 la Academia de Ciencias de París dio cuenta de las medidas geodésicas realizadas entre Dunkerque y Perpignan (Francia) por Jacques Cassini (1677-1756), quien concluye que la Tierra es un elipsoide de revolución alargado por los polos. Esta interpretación de una Tierra oblonga, pronto desechada por los científicos, incluso los más cartesianos, fue mantenida por otros autores como una reacción a la influencia de Newton en el continente.

Una primera decisión a tomar en las operaciones geodésicas era optar entre las medidas de longitudes de grados de paralelo o de meridiano (Lamontagne, 1964b). El italiano Giovanni Poleni (1683 - 1761) ganó el premio de la Academia de París en 1733 con un trabajo en el que demuestra que, para determinar la figura de la Tierra, eran más fiables las medidas hechas sobre el meridiano que sobre el ecuador. Pierre L.M. de Maupertuis (16981759), francés y newtoniano hasta el extremo de ser calificado por Voltaire como el Galileo de Francia en la batalla por introducir el newtonianismo en París, adoptó la propuesta de Poleni y la puso en práctica en la expedición geodésica a Laponia que, patrocinada por la Academia parisina, dirigió en 1736 asistido, entre otros, por Alexis Claude Clairaut (1713-1765) y el astrónomo sueco Anders Celsius (1701-1744). Un año antes, había partido para Perú otra expedición auspiciada también por la Academia con el mismo propósito de aportar datos esclarecedores en la polémica sobre la figura de la Tierra; expedición en la que, entre otros, participaron el geógrafo Charles-Marie de la Condamine (1701-1774), el profesor de hidrografía de la Universidad de Le Havre, Pierre Bouguer (1698-1758) y los cosmógrafos y marinos españoles Jorge Juan (1713-1773) y Antonio de Ulloa (1716-1795), nombrados a tal efecto por el Consejo de Indias. A pesar de los desacuerdos entre los resultados obtenidos en las expediciones a Laponia y Perú (donde hubo sonadas discrepancias entre La Condamine 
y Bouguer), la confrontación de los mismos con los realizados años anteriores en Francia hizo dudar a algunos académicos sobre la exactitud de estos últimos, lo que condujo a nuevas mediciones y expediciones (Maheu, 1966).

En las expediciones mencionadas, la medida del tiempo se hacía con el péndulo que bate segundos, ya que hasta mediados del siglo XVIII no se dispuso de relojes fiables. A tal efecto, en todas las expediciones, y en las sucesivas latitudes donde se encontraran, medían la longitud del péndulo. Asimismo, para la determinación de la vertical de un lugar, tomando como referencia las posiciones de las estrellas, se utilizaba un instrumento en el que el elemento básico era una plomada. Pero se plantearon, especialmente los expedicionarios a Perú, si una vez suspendida la plomada por su centro permanecería indefectiblemente señalando la vertical, con el fin de considerar las correcciones pertinentes tanto en la medida del tiempo como en el establecimiento de la latitud de los lugares elegidos como observatorios. La evidencia de tal desviación, en las proximidades a grandes masas, como las montañas, fue una confirmación más de la ley newtoniana de la atracción.

Admitida la influencia gravitatoria de las montañas sobre la oscilación del péndulo y sobre el peso de los cuerpos, Bouguer acometió varios experimentos para determinar la relación entre la densidad de la montaña, el Chimborazoo en su caso ${ }^{1}$, y la densidad media de la Tierra. Obtuvo dos resultados muy dispares: por una parte, que la Tierra debería ser unas cuatro veces más densa que la montaña; por otra, doce veces más (Poynting, 1894). A pesar de lo erróneo de sus resultados, es destacable la conclusión de que la Tierra fuera más densa en su conjunto que en la superficie, desestimando otras controvertidas propuestas como que la Tierra era un enorme cascarón hueco o un globo lleno de agua. (Recordemos que Newton, en la proposición x, teorema $x$ del libro tercero de los Principia conjetura que la densidad de la Tierra es cinco o seis veces la del agua). Es digno de mención el caso de Bouguer, en lo tocante a las comentadas reticencias francesas hacia las teorías newtonianas, ya que siendo un defensor de la ortodoxia cartesiana, cuando se enfrentó a la realidad y de ella hubo de extraer medidas y conclusiones, tuvo que recurrir necesariamente a los planteamientos del caballero Newton, reconociendo «la completa suficiencia del sistema de la gravitación newtoniana, lo que supone un gran triunfo para la filosofía inglesa» (Lamontagne, 1964a).

En Inglaterra, año de 1714, se formalizó el British Government's Longitude Act, que contaba con 20.000 libras destinadas a la realización de observaciones, medidas, experimentos y diseño de material para la mejora de la astronomía y la navegación. Los miembros, familiarmente conocidos como Board of Longitude, presididos por el primer lord del Almirantazgo, eran almirantes, profesores de matemáticas y astronomía de Cambridge, el Presidente de la Royal Society y el astrónomo real. En 1765 fue nombrado astrónomo real, el reverendo Nevil Maskelyne (1732-1811), quien en 1772 leyó en la Royal
Society el artículo titulado A Proposal for measuring the Attraction of some Hill in this Kingdom by Astronomical Observations $^{2}$. Si el experimento de Bouguer se repitiera, dice el reverendo, no sólo proporcionaría una prueba experimental irrefutable de la gravitación universal, como ya habían admitido los franceses, sino que también «serviría para darnos una idea mejor de la masa total de la tierra y la densidad proporcional de la materia cerca de la superficie comparada con la densidad media de la tierra en su conjunto» (Howse, 1989). Consideraba que tal experimento, además de contribuir al esclarecimiento de la controversia sobre la constitución interna de la Tierra, sería un honor para la nación que lo hiciera y para la Sociedad que lo ejecutara. El Consejo de la Royal Society puso en marcha inmediatamente un Commitee for Measuring the Attraction of Hills, compuesto por Maskelyne, Cavendish, Franklin, el honorable Daines Barrington, y el reverendo Samuel Horsley. Con esta nueva creación, los experimentos para «pesar» la Tierra, como también fueron denominados los asociados con la «atracción de las montañas», cobrarían relevancia entre los newtonianos ingleses. En el verano de 1773, el astrónomo Charles Mason (1730-1787) fue encargado de buscar una colina apropiada en las tierras altas de Escocia para la réplica del experimento de Bouguer que había propuesto Maskelyne. La montaña elegida fue Perthshire, en el centro de Escocia, llamada por los habitantes vecinos Schehallien (el nombre con que suele citarse es Schiehallion).
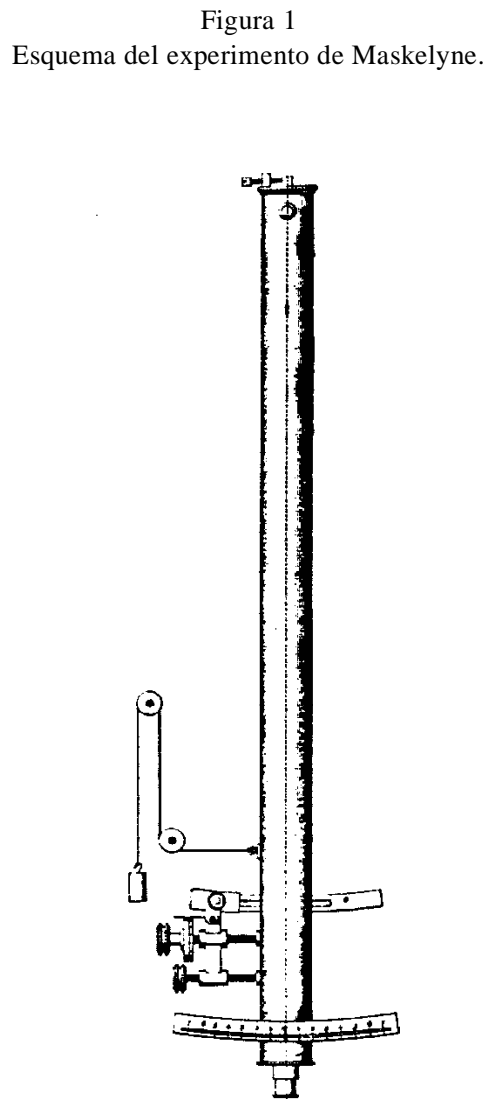

ENSEÑANZA DE LAS CIENCIAS, 2000, 18 (2) 
Sin entrar en los pormenores del experimento (Maskelyne, 1775), que se encuentra minuciosamente detallado en la biografía de Maskelyne (Howse, 1989) en artículos (Leadstone, 1974) y en libros de texto (Poynting et al., 1924; Galindo et al., 1998), la disposición de los instrumentos se hizo en oposición, en las caras norte y sur de la montaña respectivamente, como se indica en la figura 1. Las principales operaciones a realizar fueron: a) encontrar mediante observación celeste la diferencia aparente de latitud entre las dos estaciones; $b$ ) encontrar por triangulación la verdadera diferencia de latitud entre dichas estaciones; $c$ ) determinar la figura y dimensiones de la colina, de la cual, conociendo la desviación obtenida de la diferencia entre $a$ y $b$, puede determinarse la densidad media de la Tierra. El análisis de los resultados y su tratamiento matemático (Hutton, 1779) corrió a cargo de Charles Hutton (1737-1823), profesor de matemáticas de la Real Academia Militar inglesa, quien, en su informe, dice haber utilizado para resolver tan complicados cálculos matemáticos el método de análisis debido principalmente a Cavendish.

La desviación de la plomada debida a la montaña fue estimada en 12". Pero, según los cálculos, si la montaña tuviera la misma densidad que la Tierra en su conjunto, la desviación debería haber sido de 21 ", es decir 1,8 veces la cantidad observada. Hutton y Maskelyne consideraron que esa cantidad $(1,8)$ era la relación entre las densidades Tierra-montaña. A partir de muestras de la roca, estimaron su densidad como unas 2,5 veces la del agua, siendo por tanto la de la Tierra unas 4,5 veces la densidad del agua. Años más tarde, el matemático y ministro presbiteriano escocés John Playfair (1748-1812), valorando la relevancia de la densidad de la Tierra para la astronomía física, hizo un detallado estudio litológico de Schehallien, obteniendo para la densidad de la Tierra 4,713 (Playfair, 1811). El propio Hutton, a sus 84 años, publicó una revisión del trabajo hecho con Maskelyne (Hutton, 1821). También hubo intentos para medir la densidad media de la Tierra comparando el período de vibración de un péndulo en la superficie terrestre con el de otro situado en el interior de una mina. Tal es el caso de la empresa acometida en el verano de 1826 en la mina de carbón de Cornwall (Todhunter, 1876) por William Whewell (1794-1866), a la sazón profesor de mineralogía en el Trinity College de Cambridge, y por el entonces profesor de matemáticas del mismo centro, después astrónomo real, George B. Airy (1801-1892) (Ospovat, 1978).

Una vez admitida casi sin discusión la forma achatada de la Tierra, proliferaron, como vamos viendo, las medidas de su densidad para zanjar la controversia sobre su composición geológica. Laplace, que fue presidente de la Junta de Longitudes francesa, comienza así el artículo ya citado: «Uno de los puntos más curiosos de la geología es la relación entre la densidad media del esferoide terrestre y la de una sustancia conocida» (Laplace, 1904). Y dedica parte de su Mécanique céleste (1799-1825) a la figura de los planetas, refutando las ideas sobre la homogeneidad de la Tierra como bien resume en estas palabras:
«A pesar de las irregularidades que presentan los grados medidos de los meridianos, indican, sin embargo, un aplastamiento menor que el que correspondería a una Tierra homogénea; y la teoría prueba que este aplastamiento exige en las capas terrestres una densidad creciente de la superficie al centro. Paralelamente, las experiencias del péndulo, más precisas y concordantes que las medidas de los grados, indican un crecimiento de la pesantez, del ecuador a los polos, más grande que en el caso de la homogeneidad terrestre.»

\section{EXPERIMENTO CRUCIAL: EL DE CAVENDISH}

Los experimentos aludidos hasta ahora son geodésicos, astronómicos o litológicos, cuando no mentales, como el caso de Newton. La medida de la densidad de la Tierra en el laboratorio fue iniciada por Cavendish, aunque la ideación del experimento no fuera debida a él, sino al reverendo John Michell (1724-1793), astrónomo y profesor de geología en Cambridge, autor, entre otras, de las siguientes publicaciones: Conjectures concerning the cause and Observations upon the phaenomena of Earthquakes (1760), Proposal of a Method for measuring Degrees of Longitude upon Parallels of the Equator (1766), muestras de sus intereses geológicos y geodésicos. De «bella experiencia» fue calificada por Laplace (1898) la de Cavendish. Poynting (1852-1914), en The Earth: Its Shape, Size, Weight and Spin (1913), considera que el experimento fue hecho «de manera tan admirable que marca el principio de una nueva era en la medida de fuerzas pequeñas».

«El experimento para pesar el mundo o, lo que es igual, la determinación de la densidad de la tierra tenía un inmenso atractivo para Cavendish. Unía el principio de la gravitación universal - punto fijo de su filosofía newtoniana- con las ciencias de la geología y la astronomía, intereses que llegaron, muy probablemente por su amistad con Michell, a ocupar el primer plano en sus investigaciones de finales de 1780 en adelante», escribe McCormmach (1968) en un documentado artículo sobre la relación personal y científica entre aquellos dos amigos y compañeros de la Royal Society, elegidos ambos el año 1760. En la correspondencia cruzada entre ellos, se pone de manifiesto el interés por la astronomía entre 1783 y 1784 , y por la geología en 1788. La dedicación de Michell y Cavendish a los estudios gravitacionales es considerada como una destacable contribución a la visión del mundo unificado desde una perspectiva newtoniana. Además del experimento sobre la densidad terrestre, Michell diseñó un plan en 1784 para pesar las estrellas mediante el retraso gravitacional de la luz emitida por ellas, sugiriendo incluso la hipótesis de que una estrella de la misma densidad que el Sol, pero con un diámetro quinientas veces mayor, no permitiría que su luz escapase hasta el infinito. Si bien no me ocuparé de este asunto, merece ser mencionado porque estamos hablando de lo que más de una centuria después se expresó como que la «luz pesa», confirmando las predicciones hechas por Albert Einstein (1879-1955) en su principio de relatividad general, y aportando a su vez un 
anticipo de lo que hoy se conoce como agujeros negros. No obstante, respecto al interés de Cavendish por la densidad de la Tierra, hubo opiniones diversas, como la del astrónomo y meteorólogo inglés Francis Baily (17741844), quien, a juzgar por el número de experimentos realizados, sólo 23, frente a los 2.153 que hizo el propio Baily, opina que «el objetivo de Cavendish al redactar su Memoria era más la descripción de una muestra de lo que él consideraba ser un excelente método para responder a tan importante cuestión que la obtención de un resultado» ${ }^{3}$. Heilbron (1994), tras citar también este comentario de Baily, apunta que, a pesar de los resultados satisfactorios de Cavendish probando que la fuerza de la gravedad sigue la misma ley tanto para grandes como para cortas distancias, no había conseguido convencer a los contemporáneos de que todavía tenían sus dudas sobre la universalidad de la ley de Newton. En cualquier caso, el experimento de Cavendish no tardó en convertirse en una referencia paradigmática para cuantos, en lo sucesivo, se ocuparon en medir la densidad de la Tierra. El ya mencionado Airy (1834) escribe: «[...] la más notable demostración experimental de la atracción recíproca de los cuerpos consiste en una serie de experimentos hechos al final del siglo pasado por Cavendish» ${ }^{4}$.

Como dice McCormmach (1968), «pesar las estrellas y pesar el mundo eran dos proyectos ambiciosos» que contaron, a su vez, con dos protagonistas idóneos: Michell, considerado como uno de los más ingeniosos filósofos naturales del siglo xviII y Cavendish, a quien se atribuían habilidades comparables con las de Newton. «Los dos-escribe McCormmach-formaban grupo aparte de sus colegas en el dominio tanto de las matemáticas como de las ciencias experimentales». Se supone que la amistad entre ambos surgió en Cambridge. Probablemente, atraído Cavendish por la fama de «excelente filósofo» de que gozaba Michell, y ya inclinado hacia la filosofía natural, debió asistir a algunas de las lecciones del reverendo. En 1760, año de sus respectivos nombramientos como Fellows de la Royal Society, Cavendish fue admitido en el Royal Society Club, una sociedad científica muy limitada en la que sus miembros se reunía semanalmente a cenar. En sus frecuentes viajes a Londres, Michell era un huésped habitual del Club. No obstante, no hay constancia de su relación hasta más tarde, cuando se cartean durante el período 1783-88. De los diversos motivos científicos contenidos en la correspondencia destaca el gran interés compartido por los instrumentos de precisión, con la aspiración de extender los logros de Newton siguiendo los pasos de la filosofía legada por tan ilustre compatriota. Se ocuparon no sólo de las fuerzas constatadas ya como decrecientes con el inverso del cuadrado de la distancia (gravitatorias, eléctricas y magnéticas), sino también de las fuerzas de corto alcance, como muestran los trabajos no publicados de Cavendish ${ }^{5}$, investigando las propiedades matemáticas de las mismas en dinámica, calor y neumática, aunque no llegaran a pronunciarse sobre una filosofía de la materia como hoy pensamos que hubiera sido esperable y coherente.

«Cuando las investigaciones de Cavendish se contemplan en conjunto, ciertos hechos tienen una característi- ca predominancia; destaca un amplio rango de intereses, una pasión por la excatitud y un sobresaliente talento para la elección adecuada de valiosos asuntos a investigar. Aunque sus actividades se desarrollan casi todas en el siglo XVIII, su visión parecía extenderse hacia el futuro. Las palabras de Sir Edward Thorpe son significativas: "En la mayoría de sus trabajos, su tendencia de pensamiento parece haber sido dirigir el curso del subsiguiente progreso de la ciencia."»

Así termina Berry (1960a) la biografía de aquel aristócrata inglés del que, en el año de su muerte, Humphry Davy dijo: «Desde la muerte de Newton, si se me permite dar mi opinión, Inglaterra no ha padecido una pérdida científica tan grande como la de Cavendish. Como su gran predecesor, ha muerto satisfecho de años y de gloria» (Berry, 1960b).

Pero volvamos al histórico experimento: «Si su salud no le deja continuar con esto [se refiere a la construcción de un telescopio], espero que al menos le permita la más fácil y menos laboriosa tarea de pesar el mundo», así se dirige por carta (27-5-1783) Cavendish a Michell, lamentando la enfermedad de éste, en un rasgo de buen humor que, en opinión de McCormmach, es el único jocoso ejemplo conocido de su enigmática vida. Y añade Cavendish: «por mi parte, no sé si hubiera preferido oír que usted había hecho el experimento, una verdadera prueba de que ya habría terminado el gran telescopio». El experimento a que Cavendish se refiere es el plan de «pesar el mundo», que tiempo ha le había expuesto el reverendo, a realizar cuando acabara la construcción del telescopio en el que tenía gran empeño. El telescopio al fin fue terminado, y adquirido por el astrónomo William Herschel (1738-1822) a la muerte de Michell. Sin embargo, el «peso del mundo» quedaría como un legado glorioso para su contertulio, amigo y confidente, el Honorabilis Henricus Cavendish, viri Honoratissimi Domini Caroli Cavendish Filius natu maximus, como fuera inscrito en el libro de admisión del Peterhouse de Cambridge, el 18 de diciembre de 1749 , a los 18 años de edad.

La balanza de torsión que Michell construyó para medir la densidad de la Tierra, mediante la observación de la atracción entre pequeñas cantidades de materia, se basaba en un principio sugerido y usado por él hacia 1768 (Mertz, 1896-1914). Era el mismo que Charles A.Coulomb (1736-1806) empleó para medir pequeñas atracciones y repulsiones eléctricas ${ }^{6}$. Al parecer, Michell, cuando ideó su experimento, desconocía la balanza y los métodos experimentales de Coulomb, según afirma Cavendish en el artículo en el que da a conocer el célebre experimento que ha pasado a la historia con su propio nombre (Cavendish, 1798) y por casi lo único que es conocido. Asimismo, en sus trabajos de juventud, Michell, interesado por el principio newtoniano de la atracción, llegó a establecer, por primera vez (Hardin, 1966), la ley inversa del cuadrado de la distancia para la atracción y repulsión magnéticas.

A la muerte de Michell, la balanza pasó a manos del reverendo Francis John Hyde Wollaston, que desempe- 
ñaba la cátedra jacksoniana de astronomía en Cambridge y quien tiempo atrás había proporcionado datos a Cavendish para su estudio sobre la altura de la aurora boreal. No considerándose dicho profesor en condiciones de emprender el experimento de Michell, le pasó la balanza a Cavendish, quien, a partir de la de Michell, deformada y con algunos inconvenientes de diseño y de materiales, construyó otra que instaló en un edifico del jardín de su casa en Clapham, donde se dice que sólo en una ocasión entró una mujer, y por cuyos alrededores paseaba al atardecer tan extraño vecino, siempre solitario y por en medio del camino para evitar el encuentro con otros paseantes (Aykroyd, 1935).

Figura 2

Balanza de Michell y Cavendish.

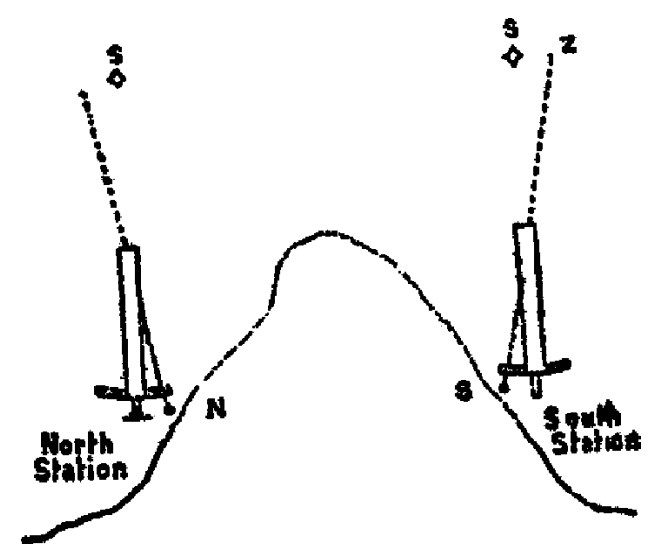

Preocupado por conseguir la mayor precisión posible en sus medidas, las modificaciones hechas iban encaminadas a evitar perturbaciones debidas, especialmente, a corrientes de aire, cuya eliminación constituía la principal dificultad del experimento, pero también a evitar cambios de temperatura, de presión, efectos magnéticos, incluso fenómenos de electrización. Para aislarla del ambiente exterior, la introdujo en un armazón de madera $G G H H G G$ (Fig. 2) y calculó el posible efecto gravitacional que aquella caja de caoba pudiera ejercer sobre las masas suspendidas. La balanza de Cavendish constaba de dos masas metálicas ligeras $x$, acopladas en los extremos de una estructura rígida de madera $\mathrm{ghmh}$, suspendida del hilo $l h$ que a lo largo del proceso experimenta la torsión. Las masas metálicas grandes $W$, externas a la balanza, ejercen la atracción gravitatoria sobre las menores $x$, pudiéndose cambiar de posición las $W$ mediante la polea $M$. Para observar los giros de la balanza empleó las fuentes de luz $L$, los espejos $n$ situados en los extremos de la barra giratoria $h m h$ y los telescopios $T$. El principio del método consiste en medir el ángulo de torsión experimentado por la barra horizontal $\mathrm{ghmh}$.

«Para determinar a partir de esto la densidad de la tierra, es necesario averiguar qué fuerza se requiere para desviar la barra un espacio dado. Michell intentó hacerlo, poniendo la barra en movimiento y observando el tiempo de sus vibraciones.»

Así plantea Cavendish su tarea, tras 17 tomas de medidas, iniciadas el 5 de agosto de 1797 y finalizadas el 30 de mayo de $1798 \mathrm{y}$, hechos los cálculos pertinentes incluidos en el apartado «On the Method of computing the Density of the Earth from these Experiments», cuyo desarrollo excede los objetivos de este trabajo ${ }^{7}$, concluyo que: «la densidad de la tierra es unas 5,48 veces mayor que la del agua».

Repetidas las medidas con otro hilo de torsión obtuvo resultados análogos.

A pesar de lo precavido que fue Cavendish en evitar perturbaciones y respetuoso con los resultados obtenidos por sus predecesores en tal empresa, concluye: «De acuerdo con los experimentos hechos por el Dr. Maskelyne, sobre la atracción de la colina Schehallian, la densidad de la tierra es 4,5 veces la del agua; que difiere bastante más de lo que yo esperaba con la determinación precedente. Pero no entraré en ninguna consideración sobre qué determinación es la más acertada hasta que no haya examinado más cuidadosamente cuánto -la determinación precedente- ha sido afectada por irregularidades cuya cantidad no he podido medir». Desconozco si Cavendish repitió las medidas, mejorando las condiciones experimentales como parecía ser su propósito. En sus Scientific Papers (nota 5), que incluyen trabajos no publicados hasta entonces (1921), no hay ninguno relativo a la densidad de la Tierra, ni quienes se han ocupado de su vida y obra mencionan que aquel propósito de revisión fuera llevado a cabo.

\section{EL ANACRONISMO: LA MEDIDA DE $G$, CONSTANTE DE LA GRAVITACIÓN UNIVERSAL}

«Newton no comprendió toda la noción de gravedad de golpe; pero fue esto lo que le indujo a emprender un largo y complejo viaje matemático que terminó con su ley de la gravitación. Incluso habiendo llegado hasta aquí, tendría que pasar un siglo antes de que el excéntrico físico Cavendish se las arreglase para determinar el valor de $G$, la constante de gravitación. Sin embargo, el que la fórmula estuviese incompleta no impidió que Newton reclamara toda la atención posible para su fórmula.» Esto se dice en una reciente publicación (Strathern, 1999), como en otras muchas de las que citaré algunas. Es decir, se afirma que Cavendish determinó el valor de la constante $G$ de la gravitación universal, llamándola en ocasiones «constante de Cavendish», cuando la realidad de su trabajo fue, como acabamos de ver, aportar un nuevo método para medir la controvertida densidad de la Tierra. Este método, de tal reputación, despertó el interés de la comunidad científica de manera que, a lo largo de los siglos XIX y Xx, han sido muchas las repeticiones del mismo, si no idénticamente, con variaciones que no desvirtuaron en lo fundamental la idea originaria $^{8}$. 
La inclusión de la constante en la expresión de las fuerzas gravitacionales newtonianas, la he encontrado por primera vez en la $4 \mathrm{a}$. edición de Cours élémentaire de Physique de Nicolás Deguin (París, 1844); la 1a. edición de 1836 no la incluye. Sin embargo, debió ser tenida en cuenta en alguna edición intermedia porque la traducción que de aquella obra hizo al castellano Venancio González Valledor, catedrático de física en los Estudios de San Isidro de Madrid, Curso elemental de Física (Madrid, 1841), utiliza la constante para calcular las densidad de la Tierra, denotándola por $f$, sin asignarle valor numérico alguno, y a la que define como «atracción de la unidad de masa a la unidad de distancia». Pero todavía no escribe la fórmula newtoniana en la forma completa que conocemos, sino de manera parcial, en la expresión $f M / R^{2}$ para lo que entonces se denominaba fuerza atractiva, simbolizada por $g$ y que corresponde a «la velocidad adquirida al cabo de la primera unidad de tiempo por un cuerpo que cae» e imprimida por la pesantez del mismo.

La escritura completa de la ley de Newton, así como la primera medida del valor de $f$, por lo que hasta ahora puedo afirmar, data de 1873, en una memoria presentada a la Academia de Ciencias de París - «Détermination nouvelle de la constante de l'attraction et de la densité moyenne de la Terre»- por Cornu y Baille (Comptes Rendus, 1873, 76 [15], pp. 954-958) ${ }^{9}$. Dicen los mencionados autores:

«Después del descubrimiento de la ley de la atracción universal por Newton, un problema experimental de una gran importancia se les planteó naturalmente a astrónomos y físicos, a saber: la determinación del valor numérico de la constante que expresa la atracción recíproca de dos unidades de masa situadas a la unidad de distancia [...] La determinación de esta constante designada por $f$ tiene un interés especial en astronomía. En efecto, la tercera ley de Kepler permite obtener directamente el valor de la masa total de dos cuerpos actuando uno sobre otro, tras el conocimiento de dos elementos de su movimiento, el semieje mayor $a$ de la órbita y el tiempo $T$ de una revolución si se conoce con precisión el valor de $f$; pues se tiene la relación ${ }^{10}$ :

$$
\frac{a^{3}}{T^{2}}=f \frac{\left(m+m^{\prime}\right)}{4 \pi^{2}}
$$

A falta de conocer esta constante con una exactitud suficiente, los astrónomos sólo determinan las relaciones entre masas de diferentes elementos del sistema solar, sea por una doble aplicación de esta fórmula a los planetas acompañados de satélites, sea mediante el cálculo de perturbaciones. El valor absoluto de las masas de los cuerpos celestes, necesaria para el conocimiento de su densidad, no es posible más que por la determinación de la masa absoluta o de la densidad media $D$ de la Tierra ligada a la constante de atracción por la fórmula:

$$
f \Delta=\frac{3}{4} \frac{g^{2}}{R} \gg
$$

En la deducción de esta expresión aplican la ley de Newton escrita ya como la igualdad de todos conocida:

$$
F=f \frac{m m^{\prime}}{r^{2}}
$$

Admitiendo que la densidad de la Tierra sea 5,48, la obtenida por Cavendish, a quienes los autores se refieren como «el ilustre físico inglés», deducen que $« f: g^{2}=0$, $0^{14} 682$ (tomando como unidades el metro y el gramo)» ${ }^{11}$.

Después de referirse, Cornu y Baille, a las medidas de la densidad de la Tierra hechas por Reich $(1838,1849)$ y Baily (1843), informan que: «la importancia de la cuestión, tanto en física como en mecánica y en astronomía, nos parece merecer un nuevo estudio, de manera que el método experimental sea aplicable no sólo a la medida de la constante de atracción sino también a un conjunto de otras determinaciones físicas [...] Hemos comenzado, por tanto, un estudio completo de la balanza de torsión, sobre todo desde el punto de vista de las medidas absolutas».

Estas medidas absolutas sólo pueden obtenerse a partir de igualdades matemáticas y no de proporciones, como Newton propuso su ley gravitacional. El misántropo Sir Henry no podía plantearse la inclusión de $G$, es decir, la fuerza gravitatoria entre unidades de masa a la distancia unidad, en la proporcionalidad newtoniana - «cerrándola»- y concluir así en la igualdad que la convierte el conocimiento de $G$, porque ésta desempeña un papel dimensional no contemplado en las leyes físicas de entonces, cuando todavía no se habían definido siquiera unidades para la magnitud fuerza.

Puesto que no es mi propósito ocuparme de la constante de la gravitación universal, simbolizada a finales del siglo XIX por $G^{12}$, y así la denominaré en lo sucesivo, sino del anacronismo que supone atribuirle a Cavendish su medida, no procede abundar en la constante misma. Me limitaré, para terminar, a hacer algunos comentarios sobre el tratamiento erróneo que suele darse al «experimento de Cavendish» en libros de texto y otras publicaciones relativas a la gravitación universal. Tampoco es necesario mostrar demasiados ejemplos de tal anacronismo; basta con echar una ojeada a los libros de física a nuestro alcance para comprobar cómo en muy pocos se menciona la medida de la densidad de la Tierra por Cavendish, y en muchos la información se da al contrario de cómo fueron los hechos, es decir, atribuyen al famoso experimento la medida de $G$ como paso previo para calcular la masa de la Tierra. «Una vez determinado el valor de $G$, puede utilizarse para hacer el cálculo de lo que se llama a menudo pesar la tierra», se lee en Holton, Roller (1972). Este experimento se ha llamado pesar la tierra. Cavendish afirmó que estaba pesando la tierra, pero lo que él medía era el coeficiente $G$ de la ley de la gravedad. Éste es el único modo en que se determina la masa de la tierra. $G$ resultó ser $6,670 \cdot 10^{-11}$ $n \mathrm{~m}^{2} / \mathrm{kg}^{2} \gg$, dicen Feynman, Leigthon, Sands (1971). «El resultado de este trabajo proporcionó la constante de la gravitación universal que completa la ley de Newton 
sobre el inverso del cuadrado de la distancia, calculando con esta constante la aceleración de la gravedad $g$, podemos encontrar la densidad media de la Tierra», escribe Segré (1983). Ejemplos como éstos pueden encontrarse en abundancia, aunque una vez entendida la doble lectura que debe hacerse de los mismos no hay que temer errores de comprensión y el anacronismo queda aclarado. Según vimos en la Memoria de Cornu y Baille, la relación entre la densidad de la Tierra y $G$ permite calcular la una si la otra es conocida ${ }^{13}$. Quizá haya sido esta fácil identificación lo que indujera a hablar casi indistintamente de ambas, prefiriendo la mayoría de los autores referirse a $G$, obviando la densidad de la Tierra. Si así fuera, y haciendo constar tal advertencia, no habría por qué preocuparse ${ }^{14}$.

Incluso, hecha la aclaración, puede resolverse la confusión aun mayor, en cuanto a la prioridad de que goza Cavendish, a que se presta la placa que las Royal Society londinense y Astronomical Society, en 1982, colocaron en Schiehallion para conmemorar el 250 aniversario del nacimiento de Maskelyne, en la que se lee, a propósito de aquel experimento: «[...] con el tiempo, éste fue llamado "la atracción de las montañas". De hecho, ésta llegó a ser la primera determinación de la constante de la gravitación universal de Newton». Ello es literalmente falso y, de haberse pretendido calcular $G$ a partir de la densidad de la Tierra obtenida de este experimento, 4,5 veces la del agua, hubiera sido desestimada frente al derivado de las medidas de Cavendish.

Aunque se asuma que hablar de densidad de la Tierra es equivalente a hablar de $G$, identificación que comparto si se deja claro el significado y momento histórico en que ambas fueron determinadas, hay casos en que, pretendiendo simplificar tanto los detalles, se transmiten informaciones inadmisibles. Por ejemplo: «Cavendish encontró para $K$ (así denota el autor la constante gravitacional)

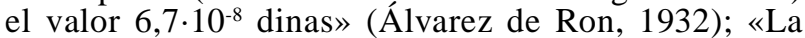
primera determinación experimental de la constante $G$ la hizo Henry Cavendish en 1798 [...] utilizó la ley de la gravitación universal y obtuvo para $G$ un valor cercano a $10^{-10} \mathrm{~N} \mathrm{~m}^{2} / \mathrm{kg}^{2}$ »(Cetto, 1991); «Él [a Cavendish se refiere] encontró que la atracción gravitatoria entre dos masas de un kilogramo separadas un metro es $6,67 \cdot 10^{-11}$ newtons. Cavendish determinó el valor de $G$ mencionada en el último parágrafo. Después del éxito de Cavendish midiendo la fuerza gravitatoria, fue posible encontrar la masa de la tierra» (Blanc, Fischler, Gardner, 1967); «Teniendo a su disposición un valor confiable para $G$, Cavendish lo empleó en la ecuación $G={ }_{g} R^{2} /{ }_{M}$ y resolvió para $M_{t}: M_{t}=g^{2}{ }_{G}$. Su resultado para la masa de la Tierra fue casi tan exacto como su medición de $G$ » (Orear, 1989).

Asimismo, se pueden proponer conclusiones igualmente anacrónicas inducidas por el anacronismo sobre la medida de $G$, tales como la siguiente: «Reconsideremos la ecuación de la gravitación de Newton a la luz de esta discusión. Cavendish, más de una centuria después de Newton, realizó un experimento para medir la fuerza de atracción entre pares de esferas metálicas y confirmó la relación $F=G^{M m} / R^{2}$. Desde este experimento, relaciona- dos mediante medidas operacionales directas los términos de la relación misma, parece razonable, pues, llamar a la relación ley (en lugar de teoría) de la gravitación de Newton»(Gardner, 1975). Esta idea, asociada con el hecho obvio, ya comentado, que la determinación de $G$ completa la expresión de la ley de la gravitación, homogeneizando las dimensiones de la igualdad, puede ser cierta en sí misma, si se admiten las premisas propuestas por Gardner sobre la distinción entre leyes y teorías, pero está desubicada en el tiempo. Y esto es un error que debe aclararse. Situar en el mismo tiempo asuntos que no fueron coincidentes y que ni siquiera era propicio plantearse es un falseamiento de la historia que, como vemos, a su vez, multiplica los errores; errores que pueden trastocar, como en el caso que nos ocupa, aspectos epistemológicos de las ciencias físicas.

\section{ESCOLIO}

A propósito del anacronismo expuesto, detectado durante la búsqueda de documentación para dar una orientación histórica a la unidad didáctica «Interacción gravitatoria» del libro Física $2^{\circ}$ Bachillerato LOGSE antes mencionado, son pertinentes algunos comentarios.

En primer lugar, echemos un breve vistazo a la consideración que de la historia de la ciencia se ha tenido y viene teniéndose en las enseñanzas científicas. Aunque sea casi anecdótico, conviene apuntar que el Plan Pidal de 1845, redactado por Gil de Zárate, Revilla y Guillén, políticos afines a la ideología liberal progresista, incluyó Historia de las Ciencias como asignatura obligatoria de doctorado. Recordemos que de dicho plan procede la creación de los institutos de segunda enseñanza y que fue tenido en su tiempo, y así ha pasado a la historia, como uno de los más avanzados en el proceso de secularización de las enseñanzas ${ }^{15}$. Cierto es que este caso, relacionado con la estimación curricular de la historia de la ciencia, es puntual como algunos otros que le sucedieron. Más sólida fue la propuesta hecha en los Cuestionarios de los Cursos del Grado Profesional de las Escuelas Normales del Magisterio Primario (Boletín, 1932), en la que a propósito del Cuestionario de Metodología de las Matemáticas, se dice:

«Tampoco puede prescindirse de incluir algo sobre historia de la ciencia que estudiamos, ya que la evolución experimentada por la matemática a través de los siglos ha sido la misma que va sufriendo en nuestro espíritu según la vamos adquiriendo; estimando, por tanto, el concimiento de la historia de la matemática, además de un grado de ampliación de cultura en nuestros alumnos, una base precisa para el estudio de su metodología».

Esta disposición desciende del nivel doctoral antedicho al de la formación de los maestros, y después llegará al del alumnado primario y secundario como puede verse, por ejemplo, en los programas renovados de EGB (1981) o en los actuales diseños curriculares propuestos en la LOGSE, sobre todo para bachillerato. 
Acercándonos a tiempos más recientes, y con una visión más amplia, recordemos algunas aportaciones. A las publicaciones en forma de diálogos o confesiones al estilo galileano, en las que se daba a conocer aspectos históricos y filosóficos de las ciencias, sucedieron los más formales y estructurados libros de texto o de consulta en el aula, en los que lo histórico era objeto de estudio y exigido en los correspondientes exámenes. Caben reseñarse: Hogben, L. (1938). Science for the Citizen A Self-Educator based on the Social Background of Scientific Discovery, Londres: George Allen; Taylor, L.W. (1941). Physics. The Pioneer Science, EEUU: The University Press Cambridge; Swenson, H.N. y Woods, J.E. (1957). Physical Science for Liberal Arts Students, Nueva York: John Wiley. Hay que hacer una mención especial de las obras de James B. Conant (1893-1978), On Understanding Science: An Historical Approach (1947) y Harvard Case Histories in Experimental Science (1957), escrita esta última en colaboración con Nash y los Roller, coautores éstos con Gerald Holton de Fundamentos de la física moderna (1958), considerada una revisión del libro de Holton, Introducción a los conceptos y teorías de las ciencias físicas (1952), ambos editados en castellano y sobradamente conocidos por el profesorado español interesado en la historia de la ciencia. Todas estas obas son antecedentes del Harvard Project Physics, que, a partir de 1964, Rutherford, Holton y Watson inician, dándolo a conocer en 1970 con los siguientes propósitos: $a$ ) diseñar un curso de física con orientación humanística; $b$ ) desarrollar un curso que atrajera a los estudios introductorios de física a gran número de estudiantes de la educación secundaria superior; y c) contribuir al conocimiento de los factores que influyen en el aprendizaje de la física». Estos objetivos fueron obviamente compartidos por quienes anduvieran indagando en cómo canalizar las enseñanzas científicas desde una perspectiva histórica.

Una de las primeras evaluaciones hechas a la aplicación de los casos-estudio históricos en la enseñanza de las ciencias es The Use of Case Histories in the Development of Student Understanding of Science and Scientists, que Leopold E. Klopfer y William W. Cooley elaboraron en 1961, en la Universidad de Harvard, y presentaron como mérito para conseguir un contrato en la United States Office of Education. Concluían aquéllos, entonces principiantes: «el método es definitivamente efectivo para incrementar en los estudiantes el conocimiento de la ciencia y de los científicos cuando es aplicado a las clases de biología, química y física de la educación secundaria superior». En cuanto a los resultados de las primeras aplicaciones del proyecto Harvard, fueron publicados en A Case Study in Curriculum Evaluation: Harvard Projecto Physics, que, si bien hasta la fecha no he tenido ocasión de consultar, sí los comentarios sobre el mismo hechos por Welch y Walberg (1972). Estos autores comparan los cursos de física tradicionales con los proyectos PSSC y Harvard, a partir de la evaluación de estos últimos. En general, tanto los tradicionales como el PSSC son calificados como más dificultosos y, en cierto modo, más aburridos que el Harvard, para el que «los estudiantes califican la física como más histórica, humanística, filosófica, social, ar- tística y menos matemática y aplicada que la física vista en los otros cursos». Conclusión que, a su vez, obliga a prevenir el riesgo de que la perspectiva histórica no suponga una pérdida del carácter conceptual, teorético, experimental, aplicado e, ineludiblemente, matemático de la física.

Muestra del interés por la utilización didáctica de la historia de la ciencia, caben destacar, más recientemente, las actividades desarrolladas desde el Interdivisional Group on the History of Physics of the European Physical Society y el International History, Philosophy and Science Teaching Group, que periódicamente celebran congresos cuyos Proceedings dejan constancia de las múltiples intervenciones en la dirección que nos ocupa. Obra en mi opinión de consulta obligada, tanto por el contenido propiamente dicho como por la profusa información bibliográfica, es la de Matthews (1994), así como la recopilación de artículos hecha por Shortland y Warwick (1989). Asimismo hay que citar la acogida prestada a la historia de la ciencia por publicaciones periódicas como Enseñanza de las Ciencias, Alambique o Science \& Education. Contributions from History, Philosophy and Sociology of Science and Mathematics.

Ahora bien, a pesar de las contribuciones hechas hasta ahora en el tratamiento didáctico de la historia, la filosofía y la sociología de la ciencia, las aportaciones siguen siendo aisladas y poco integradas en el proceso real al que han de ajustarse profesores y alumnos en el aula. Una primera exigencia que habría de contemplarse es el acercamiento lo más riguroso posible al hecho histórico en cada momento. Destaco este requisito, entre otros que habría de señalar, de los que no me ocupo en esta ocasión, para avalar desde un punto de vista didáctico, y no meramente histórico, el trabajo aquí expuesto. El anacronismo con que se viene procediendo, especialmente en los libros de texto, respecto a la introducción de la constante de la gravitación universal en la ley de Newton, es una muestra, acaso infrecuente, pero ahí está, de lo que supone una tergiversación de la historia ${ }^{16}$. Para evitar tales sucesos sería recomendable la contrastación, o al menos cierta cautela, respecto a las referencias históricas por quienes pretenden instrumentar las actividades docentes acercándose al proceso de configuración de la ciencia como actividad humana y, por tanto, social, con unas características de racionalidad específicas y otras hasta ahora casi consideradas marginales de lo que por ciencia ha venido entendiéndose ${ }^{17}$.

Es obvio que tal rigor histórico no siempre está al alcance de quien lo pretende; incluso, para quienes lo tengan más accesible, es una tarea dificultosa. Por esto sería benéfico, para apoyar los programas de investigación didáctica con esta orientación así como para el diseño de actividades de enseñanza reglada, contar con materiales por ahora escasos o inexistentes, sobre todo en los países de habla hispana. La traducción de obras científicas fundamentales, experimentos cruciales, lecturas históricas, biografías sensatas (no las hagiografías tan próximas a una deformante beatería en la ciencia y los científicos), así como de estudios y otras publicaciones sobre la tan traída y llevada interrelación ciencia- 
sociedad, todavía en trance de identificación como disciplina o lo que sería más deseable como ámbito de conocimiento, supone una fuente de recursos imprescindible cuya carencia imposibilita la visión de la ciencia con la complejidad que le es propia, a la vez que sigue manteniendo la formación de la ciudadanía al margen de que la ciencia se tiene que considerar también como hecho cultural.

\section{NOTAS}

${ }^{1}$ Bouguer en La figure de la Terre (1749) da cuenta de sus observaciones y medidas hechas en el Chimborazo sobre el fenómeno denominado atracción de las montañas. La Condamine había hecho lo propio en Lettre à M. Dufay sur les observations faites à Chimborazo, Montagne de la Province de Quito, pour reconnaître par Expérience l'effect de l'attraction Newtonienne (1738).

${ }^{2}$ Phil. Trans., 65, pp. 495-499. Maskelyne ya se había ocupado años antes de las noticias relativas a las medidas del arco de meridiano. En «Introduction to the following Observations, made by Messieurs Charles Mason and Jeremiah Dixon, for determining the Length of a Degree of Latitude, in the Provinces of Maryland and Pennsylvania, in North America» (Phil. Trans. 58, 1768, pp. 270-328), además de los comentarios sobre aquellas observaciones geodésicas, incluye un post scríptum sobre las posibles equivalencias entre las unidades de medida inglesas y francesas, informa de los resultados obtenidos en las expediciones a Laponia, Perú y Cabo de Buena Esperanza y los obtenidos en Francia e Italia. Termina sus comentarios a propósito de las diferencias existentes con una alusión al honorable Mr. Henry Cavendish, quien estudiándolos con detalle está de acuerdo en que las medidas «pueden estar sensiblemente afectadas por la atracción de las montañas». (Cope, Robinson, 1952)

${ }^{3}$ Baily (1824) da cuenta de las extremas precauciones a tomar en el uso de la balanza de torsión, algunas expuestas por Cavendish, para obtener resultados fiables. Considerados 2004 experimentos de los realizados, estima en 5,67 el valor más aceptable para la densidad media de la Tierra. Una corrección a las medidas de Baily particularmente interesantes, no por las medidas en sí (para los fines que me he propuesto en este trabajo), sino por quienes las realizaron y por lo que tienen que ver con el anacronismo al que me referiré seguidamente, está expuesta en Cornu y Baille (1878).

${ }^{4}$ Airy (1857) comenta los resultados de sus experimentos. Sobre los resultados dispares de estas mediciones (entre 4,7 y 6,6) da su versión el capitán Jacob (1857). También cabe destacar en las medidas de la densidad de la Tierra al profesor de filosofía natural de la Academia de Minas de Freyberg (Saxony), el mineralogista Ferdinand Reich (1799-1882), quien dice haber seguido el método de Cavendish instalando la balanza en una sótano de la Academia de Minas (Reich, 1837).

${ }^{5}$ Cavendish, H. (1921). Scientific Papers, vol. II, Chemical and Dynamical, Edward Thorpe (ed.) Cambridge University Press. Este volumen contiene los trabajos no publicados de Cavendish según los manuscritos originales en posesión del duque de Devonshire, comentados por Joseph Larmor (matemáticas y dinámica), Archibald Geikie (geología), Frank W. Dyson (astronomía) y Charles Chree (magnetismo). El vol. I, The Electrical Researches, publicado el mismo año, es la edición revisada por J. Larmor de la hecha por James Clerk Maxwell en 1879. Maxwell (1831-1879) fue el primero en desempeñar una cátedra de física experimental en la Universidad de Cambridge, donde organizó el célebre laboratorio Cavendish, contando con una considerable fortuna donada por aquel excéntrico inglés. Sobre sus peculiares vida y obra, he podido consultar, aparte de otras notas biográficas menores: Berry (1960), Young (1921), Cuvier (1961), Jaffe (1931) y Crowther (1962).

${ }^{6}$ Las primeras investigaciones de Coulomb sobre la torsión datan de 1777, en una Memoria premiada por la Academia de Ciencias de París. En 1781, elegido miembro de la Academia, pronuncia su discurso de ingreso sobre torsión y elasticidad de hilos metálicos. Es en 1785 cuando da a conocer, en otra Memoria leída en la Academia, su conocida ley: «Constrution et usage d'une Balance électrique, fondée sur la propriété qu'ont les Fils de métal, d'avoir une force de réaction de Torsion proportionelle à l'angle de Torsion [...]» (Gillmor, 1971; Crowther, 1962). Cavendish, con el propósito de describir los fenómenos electrostáticos mediante modelos mecánicos, al estilo newtoniano, estableció la ley inversa del cuadrado de la distancia para las atracciones y repulsiones eléctricas, investigaciones desconocidas hasta su publicación por Maxwell (nota 8). Un anticipo de su interés por tales fenómenos es el artículo «An Attempt to Explain some of the Principal Phaenomena of Electricity by means of an Elastic Fluid», Phil. Trans., 61 (1771), pp. 584-677. Dan cuenta de estos hechos, por ejemplo: Bauer, 1949; Dorling, 1974.

${ }^{7}$ De las publicaciones en castellano, una forma simplificada de conocer la teoría de la torsión aplicada por Cavendish se encuentra en el texto Física $2^{\circ}$ Bachillerato LOGSE (Galindo et al., 1998); y desarrollado con más detalle, incluso con una parte del trabajo original de Cavendish traducido al castellano, en García Sanz, J.J. (1998), «El experimento de Cavendish», Revista de la Facultad de Ciencias, 1, Madrid, UNED.

${ }^{8}$ Para dar una idea del crédito adquirido por el experimento de Cavendish citaré otros, realizados en el siglo XIX, con el mismo fin y utilizando la balanza de torsión: 1837, Reich; 1841, Baily; 1842, Reich; 1872, Cornu y Baille; 1894, Boys; 1894, Braun; 1896, Eötvös; 1901, Burgess. Algunos han sido comentados en este trabajo. Asimismo, reproduciendo el experimento de Cavendish o introduciendo las modificaciones pertinentes, se hicieron, y continúan haciéndose, medidas de $G$, la constante de la gravitación universal. De muchas de estas medidas se da cuenta en Proceedings of the Second International Conference held at the National Bureau of Standars-«Precision Measurement and Fundamental Constans II- editados por B.N.Taylor y W.D. Phillips (1984), Washington, EEUU, Government Printing Office; también en Kestenbaum (1998) y Schwarz y otros (1998).

${ }^{9}$ El óptico Marie Alfred Cornu (1841-1902), formado en l'École Polytechnique y luego profesor de la Escuela de Minas de París, se ocupó junto con Baille de la balanza de torsión en varias ocasiones: Étude de la résistence de l'air dans la balance de torsion, C.R., 1878, 86 (9), en el que insisten en las medidas 
que es preciso tomar para evitar «influencias accesorias» en la medida de la densidad media de la Tierra por el «método de Cavendish»; Influence des termes proportionels au carré des ècarts, dans le mouvement oscillatoire de la balance de torsion, C.R., 1878, 86 (16).

${ }^{10}$ Ésta es la llamada actualmente ley armónica de Kepler o ley 1-2-3, que suele escribirse como $G\left(m_{1}+m_{2}\right)^{1}=\omega^{2} a^{3}$, para la relación que existe en el movimiento elíptico entre el semieje mayor $(a)$ de la cónica del movimiento relativo de dos masas $m_{1}$ y $m_{2}$ y el período orbital $T=2 \pi / \omega$.

${ }^{11}$ Los autores insertan esta nota a pie de página: «En efecto, si se aplica la ley de Newton a dos cuerpos cualesquiera, de masas $m, m^{\prime}$, se tiene $F=f m m^{\prime}: r^{2}$. Si uno de ellos no es otro que la Tierra, se tiene $p^{\prime}=f \mathrm{M} p^{\prime}: g r^{2}$ o $f=g \mathrm{R}^{2}: \mathrm{M}$. Comparando la masa $M$ de la Tierra con la de un volumen igual de agua y llamando $\Delta$ a la cantidad así obtenida, se encuentra la fórmula citada». En la comparación, identifican el volumen de agua con el peso del mismo, siendo, por tanto, $\Delta=M: V / g=M: 4 \pi R^{3}$ $13 \mathrm{~g}$. Para obtener la relación $f: g^{2}=0,0^{14} 682$, hay que tomar la densidad de la Tierra obtenida por Cavendish como $\Delta=5,48$ - $10^{6} \mathrm{~g} / \mathrm{m}^{3}$. El valor de $\mathrm{g}$ en París, dado en el libro de Deguin citado en el texto, es de $9^{\mathrm{m}}, 8088$ (téngase en cuenta que todavía no se habían introducido las unidades de medida en la forma dimensional adoptada a finales del siglo XIX). Cornu y Baille se refieren a una «nueva determinación de la constante de atracción». Desconozco a qué medida anterior puedan referirse, ni por quién fuera hecha, ya que ellos en la Memoria no dan ninguna cita al respecto. Una historia sucinta de las más de 20 medidas de $G$ hechas en el laboratorio, hasta hoy, pueden consultarse en Chen and Cook (1993).

${ }^{12}$ Con esa notación aparece en Poynting (1892). Seguramente la notación es anterior, pero no he tenido ocasión de consultar las referencias que Poynting hace sobre: Jolly, Wiedemann's Annalen, vol. 5, p. 112 y vol. 14, p. 331; Koening and Richraz, Nature, vol. 31, pp. 260 y 475; y sobre una «reciente» publicación de C.V. Boys de la que no da referencia. Boys utiliza la notación de $G$ en una publicación posterior a la de Poynting, en la que compara los resultados obtenidos por él mismo en las determinaciones de la densidad de la Tierra y de $G$ con los dados por Cornu y Poynting (Boys, 1895). Poynting ya había utilizado una balanza química, construida en Londres por Oertling, con el mismo propósito (Poynting, 1879).

${ }^{13}$ Poynting en el artículo citado (nota 12) utilizó para sus experimentos una balanza común, instalada primero en el laboratorio Cavendish de Cambridge, «gracias a la bondad del profesor Clerk Maxwell», pasándola después al Mason College, en Birmingham, donde finalizó las investigaciones. Los fundamentos teóricos del experimento se basan en la siguiente relación para la densidad media $\Delta$ de la Tierra: $\Delta=g R^{2} / G V$, siendo $R$ y $V$ el radio y volumen de la Tierra, respectivamente. La obtención de esta relación es inmediata, como lo es la siguiente para obtener $G$ a partir de $\Delta$. La ley de la gravitación universal para la fuerza gravitatoria entre la Tierra y un cuerpo en su superficie de masa $m$ es $F=G M m / R^{2}$, sustituyendo la masa $M$ de la Tierra en función de la densidad $\Delta$, resulta $F=4 /$ $3 G \pi \Delta m$, expresión equivalente a la del peso del cuerpo, $p=m g$, de donde: $G=3 g / 4 \pi \Delta R$. En el libro de texto (Poynting, Thomson, 1924), se desarrolla un caso numérico del que los autores concluyen: «Este ejemplo muestra que los dos problemas, la determinación de la constante de gravitación $G$ y la determinación de la densidad media de la tierra, son prácticamente el mismo.» En el apartado «The Cavendish Experiment»,pp. 36-39, los autores muestran una simplificación muy fidedigna del procedimiento de cálculo de la densidad de la Tierra seguido por Cavendish.
${ }^{14}$ Algunos ejemplos en los que se hace constar tal advertencia son: texto y artículo citados en nota 16; apartado 8.2.4., «Cavendish y el peso de la Tierra», de Fernández-Rañada (ed.) (1993); y más recientemente en Lally (1999), quien tras aclarar el anacronismo expuesto, termina así: «En cualquier caso, está claro que todos los que consideran a Cavendish el primero en calcular $G$ (o la masa de la Tierra) simplemente no han leído su trabajo. El año 1798 no era todavía el tiempo apropiado para hacer tales cálculos».

${ }^{15}$ Moreno (1988) analiza el proceso de secularización de las enseñanzas en España, donde el dificultoso sacudimiento del estilo escolástico con que se trataban en las aulas la filosofía y los escasos contenidos de física afectó sobremanera la modernización de la actividad científica, tanto académica como investigadora.

${ }^{16}$ A propósito del centenario del «descubrimiento del electrón», Lelong (1998) ha tratado de clarificar otra falsa interpretación histórica de lo que el autor llama «el mito fundador del electrón».

${ }^{17}$ Izquierdo, Sanmartí y Espinet (1999), a propósito del cambio experimentado actualmente en el modelo tradicional de ciencia, para el que método científico, cargado de una alta dosis de espíritu baconiano, se identificaba con racionalidad y ésta a su vez con la ciencia, como el más poderoso atributo de la mente, dicen: «Estos cambios de enfoque han afectado el concepto de racionalidad científica y de método científico y por ello han emergido nuevos modelos de ciencias que se refieren a la racionalidad moderada, contextual o hipotética para explicar cómo impulsan los científicos el proceso de creación científica. Este nuevo modelo de racionalidad destaca el aspecto humano, tentativo y constructivo de las ciencias.» Es por aquella presunta racionalidad infalible que se recurre a la cientificación, por ejemplo, de mensajes publicitarios para captar compradores incautos y creyentes en: «iEl frotar se va acabar! Wipp progress con oxígeno activo», «Anticaída Dercos con aminexil actúa contra la fibrosis capilar» y tantos otros ejemplos basados en la ignorancia del consumidor, persuadido de que lo tocado por la mano «casi divina» de la ciencia garantiza la bondad del producto. O lo que todavía es aún más inquietante, la arrogancia de algunos intelectuales célebres promíscuos a «la mistificación, el lenguaje deliberadamente oscuro, la confusión de ideas y el mal uso de conceptos científicos» que (Sokal, Bricmont, 1999) denuncian como una parodia «bastante frecuente en círculos posmodernos y de estudios culturales». Parodia que nos trae a la memoria aquel divertimento que el coronel José de Cadalso (1741-82) publicara en Madrid (1772) en «obsequio de los que pretenden saber mucho, estudiando poco»: Los eruditos a la violeta o curso completo de todas las ciencias, dividido en siete lecciones para los siete días de la semana o La derrota de los pedantes, publicada en 1789 por Leandro Fernández de Moratín (17601828), «sátira contra los zafios ingenios que por entonces monopolizaban las tablas de la escena y los estantes de las librerías». Ante hechos similares, en cualquier época se ha propuesto, como solución, la práctica de una adecuada ciencia escolar, en las diversas formas que a lo largo de los tiempos se ha entendido por tal.

* Este trabajo ha sido realizado gracias a la ayuda a la investigación PR 95-084, concedida por la DGICYT (1985) para una estancia en la Universidad de New South Wales, Sydney (Australia). 


\section{REFERENCIAS BIBLIOGRÁFICAS}

AIRY, G.B.(1857). Account of PendulumExperiments undertaken in the Harton Colliery for the purpose of determining the Mean Density of the Earth. Proceedings R.S.L., 8, pp. 13-18.

AIRY, G.B. (1893). Gravitazione. Milano: Manuali Hoepli. Traducción al italiano de la 1a. edición inglesa (1834, Londres, Charles Knight, 22, Ludgate Street).

ÁLVAREZ de RON, E. (1932). Nociones de física. Madrid: Instituto Samper, p. 103.

AYKROYD, W.R. (1935). The Honourable Henry Cavendish, cap. VIII de Three Philosophers (Lavoisier, Priestley, Cavendish), pp. 71-78.

BAILY, F. (1842). An Account of some Experiments with the Torsion-rod, for Determining the Mean Density of the Earth. Monthly notices of the Royal Astronomical Society, 5(24), pp. 197-206. Londres.

BAUER, E. (1949). L'Électromagnétisme hier et aujourd'hui. París: Albin Michel.

BERRY, A.J. (1960 a). Henry Cavendish. His Life and Scientific Work. Londres: Hutchinson.

BLANC, S.S., FISCHLER, A.S. y GARDNER, O. (1967). Modern Science: Forces, Change, and the Universe, p.132. Nueva York: Holt, Rinehart and Winston, Inc.

Boletín Oficial del Ministerio de Instrucción Pública y Bellas Artes, 10-11 (1932). Madrid.

BOWKER, G. (1995). In Defence of Geology: The Origins of Lyell's Uniformitarianism, en Serres, M. A history of Scientific Thought. Oxford: Blackwell Publishers Ltd.

BOYS, C.V. (1895). On the Newtonian Constant of Gravitation. Phil. Trans., 186, pp. 1-72

CAVENDISH, H. (1798). Experiment to Determine the Density of the Earth. Phil. Trans., 88(2), pp. 469-526.

CETTO, A.M. et al. (1991). El mundo de la física, vol. I, p. 286. México: Trillas.

CONDAMINE, C. M. DE LA (1986). Viaje a la América Meridional por el río de las Amazonas. Estudio sobre la quina, presentación de Lafuente, A. y Estrella, E. «Mundo Científico». Barcelona: Editorial Alta Fulla.

COPE, T.D. y ROBINSON, H.W. (1952). Charles Mason, Jeremiah Dixon and The Royal Society. Notes and Records of The Royal Society, 9, pp. 72-74.

CORNU, A. y BAILLE, J.B. (1878). Sur la mesure de la densité moyenne de la Terre. Comptes rendus, 86(11), pp. 699-702.

CROWTHER, J.G. (1962). Scientifics of the Industrial Revolution (Black, Watt, Priestley, Cavendish). Londres: The Cresset Press.

CUVIER, G. (1961). Henry Cavendish, en Eduard Farber (ed.), Great Chemists. 2 vols. París: Interscience.

CHEN, Y.T. y COOK, A. (1993). Gravitational Experiments in the Laboratory. Cambridge: University Press.

DORLING, J. (1974). Henry Cavendish's deduction of the electrostatic inverse square law from the result of a single experiment. Studies in History and Philosophy of Science, 4(4), pp. 327-348.

FERNÁNDEZ-RAÑADA, A.(ed.) (1993). Física básica, tomo I, pp. 283-288. Madrid: Alianza.
FEYNMAN, R., LEIGTHON, R.B. y SANDS, M. (1971). The Feynman. Lectures of Physics, vol. I, p. 7-13. México: Fondo Educativo Interamericano.

GALINDO, A., MORENO, A., BENEDÍ, A. y VARELA, P. (1998). Física, $2^{\circ}$ Bachillerato LOGSE. Madrid: McGrawHill, p. 296.

GARDNER,P.L.(1975). Science and the Structure of Knowledge, en The Structure of Science Education. Australia: Longman, p. 21. La discusión aludida en el texto se refiere a las condiciones en que es apropiado hablar de leyes o de teorías.

GILLMOR, C.S. (1971). Coulomb and the Evolution of Physics and Engineering in Eighteenth-Century France. Nueva Jersey: Princeton University Press.

GREENBERG, J.L. (1995). The state of the problem of the earth's shape in the 1720s: Stalemate, en The Problem of the Earth's Shape from Newton to Clairaut. Cambridge: University Press.

HARDIN, C.L. (1966). The Scientific Work of the Reverend John Michell. Annals of Science, 22, pp. 27-47.

HEILBRON, J.L. (1994). On Coulomb's electrostatic balance, en Christine Blondel y Matthias Dörries (ed.), Restaging Coulomb. Usages, controverses et réplications autour de la balance de torsion, pp. 151-161. Florencia: Leo S. Olschki.

HOLTON, G. y ROLLER, H.D. (1972). Fundamentos de la física moderna, p. 207. Barcelona: Reverté.

HOWSE, D. (1989). Nevil Maskelyne. The Seaman's Astronomer, p. 131 Cambridge: University Press.

HUTTON, C. (1779). An Account of the Calculations Made from the Survey and Measures Taken at Schehallien, in Order to Ascertain the Mean Density of the Earth. Phil. Trans., 68, pp. 689-788.

HUTTON, J. (1795). Theory of the Earth. Edimburgo: messrs. Cadell, junior, and Davies, London; and William Creech.

HUTTON, C. (1821). On the mean density of the Earth. Phil. Trans., pp. 276-292.

HUYGENS, C. (1992). Traté de la lumière. Introducción de Michel Blay). París: Dunod.

JACOB, W.S. (1857). On the Causes of the great Variation among the different Measures of the Earth's Mean Density. Proceedings R.S.L., 8, pp. 295-299.

IZQUIERDO, M., SANMARTÍ, N., y ESPINET, M. (1999). Fundamentación y diseño de las prácticas escolares de ciencias experimentales. Enseñanza de las Ciencias, 17(1), pp. 45-59.

JAFFE, B. (1931). Cavendish. A Millionaire Misanthrope turns to the Elements. Crucibles. The Lives and Achievements of the Great Chemists. Londres: Jarrolds.

KESTENBAUM, D. (1998). Gravity Measurements Close in on Big G. Science, 282, pp. 2180-2181.

LAFUENTE, A. y DELGADO, A.J. (1984 a). La geometrización de la tierra: observaciones y resultados de la expedición geodésica hispano- francesa al virreinato del Perú (1735 1744). Cuadernos Galileo de Historia de la Ciencia, Madrid: Consejo Superior de Investigaciones Científicas.

LALLY, S.P. (1999). Henry Cavendish and the Density of the Earth. The Physics Teacher, 37, pp. 34-37. 
LAMONTAGNE, R. (1964a). La Vie et l'Oeuvre de Pierre Bouguer. París: Presses Universitaires de France.

LAMONTAGNE, R. (1964b). Carta de Bouguer a Euler fechada en París (4-5-1751). op. cit., p. 226.

LAPLACE, P.S. (1773). Examen du principe de la gravitation universelle, en Sur le principe de la gravitation universelle et sur les inégalités des planètes qui en dépendent. Mémoires de l'Académie Royale des Sciences de Paris, tomo VIII.

LAPLACE, P.S. (1898). Mémoire sur la figure de la terre, leída en la Academia el 4-8-1818, en Oeuvres Complètes. París: Gauthier-Villars.

LAPLACE, P.S. (1904). Sur la densité moyenne de la Terre, en Ouvres Complètes, tomo XIII, Académie des Sciences. París: Gauthier-Villars.

LEADSTONE, G.S.(1974). Maskelyne's Schehallien experiment of 1774. Physics Education, 9, pp. 452-458.

LELONG, B. (1998). Nadie descubrió el electrón. Mundo Científico, 186, pp.71-75.

MAHEU, G. (1966). La vie scientifique au milieu du XVIII siècle: Introduction à la publication des lettres de Bouguer à Euler. Revue d'Histoire des Sciences et des leurs applications. París: Presses Universitaires de France.

MASKELYNE, N. (1775). An Account of Observations on the Mountain Schehallien for finding its Attraction. Phil. Trans., 65 , pp. 500-542.

MATTHEWS, M.R. (1994). Science Teaching. The Role of History and Philosophy of Science. Nueva York: Routledge.

McCORMMACH, R. (1968). John Michell and Henry Cavendish: Weighing the Stars. The British Journal for the History of Science, 4(14), pp. 126-155.

MERZ, J.T. (1896-1914). A History of European Thought in the Nineteenth Century, p. 320.

MORENO, A. (1988). Una ciencia en cuarentena. La física académica en España (1750-1900). Madrid: Consejo Superior de Investigaciones Científicas.

NEWTON, I. (1982). Principios matemáticos de la filosofía natural y susistema delmundo, edición de Antonio Escohotado. Madrid: Editora Nacional.

OREAR, J. (1989). Física. México: Limusa. p. 103.

OSPOVAT, A.M. (1978). Four hitherto unpublished geological lectures given by Sir Humphry Davy in 1805 from manuscripts belonging to the Royal Geological Society of Cornwall. Cornwall: The Royal Geological Society.

PALACIOS, J. (1964). Análisis dimensional. Madrid: EspasaCalpe.
PLAYFAIR, J. (1811). Account of a Lithological Survey of Schehallien, made in order to determine the specific Gravity of the Rocks which compose that Mountain. Phil. Trans., 102, pp. 347-378.

POYNTING, J.H. (1879). On a method of using the Balance with great delicacy, and on its employment to determine the Mean Density of the Earth. Proceedings R.S.L., 28 pp. 2-35.

POYNTING, J.H. (1892). On a Determination of the Mean Density of the Earth and the Gravitation Constant by means of the Common Balance. Phil. Trans., 182, pp. 565-656.

POYNTING, J.H. (1894). A History of the Methods of Weighing the Earth. Birmingham Phil. Soc. Proc. 9, pp. 1-23. Recogido en J.H.P. (1920), Collected Scientific Papers by... Cambrigde: University Press.

POYNTING, J.H. y THOMSON, J.J. (1924). Properties of Matter. A Text - Book of Physics, vol. I, 10a. ed. Londres: C. Griffin.

REICH, F. (1837). Extrait d'un Mémoire de REICH, M. sur la densité de la Terre. Comptes Rendus, 5, pp. 697-700.

ROSSI, P. (1997). El nacimiento de la ciencia moderna en Europa. Barcelona: Crítica.

SCHWARZ, J.P. et al. (1998). A Free-Fall Determination of the Newtonian Constant of Gravity. Science, 282, pp. 22302234.

SEGRÉ, E. (1983). Dalla caduta dei gravi alle onde elettromagnetiche. Personaggi escopertenella Fisica Classica, pp.153-154. Milán: Mondadori.

SHORTLAND, M. y WARWICK, A. (eds.) (1989). Teaching the History of Physics. Oxford: The British Society for the History of Science, Basil Blackwell.

SOKAL, A. y BRICMONT, J. (1999). Imposturas intelectuales. Barcelona: Paidós.

STRATHERN, P. (1999). Newton y la gravedad. Col. «Los científicos y sus descubrimientos», p. 28. Madrid: Siglo XXI.

TATON, R. (1988). Forma de la Tierra, en La ciencia moderna (1450-1800), vol. II de Historia General de las Ciencias. Barcelona: Ediciones Orbis.

TODHUNTER, I. (1873). A History of the Mathematical Theories of Attraction and the Figure of the Earth, from the Time of Newton to that of Laplace, 2 vols. Londres: Macmillan.

WELCH, W.W. y WALBERG, H.J. (1972). A National Experiment in Curriculum Evaluation. American Educational Research Journal, 9, pp. 373-383.

YOUNG, T. (1921). Life if Cavendish, vol. I de Scientific Papers (nota 5).

[Artículo recibido en junio de 1999 y aceptado en diciembre de 1999.] 